\title{
MATHEMATICAL MODELING OF FINITE TOPOLOGIES
}

\author{
MonabBati S.E. ${ }^{1}$, TORABi H. ${ }^{2}$
}

\begin{abstract}
Integer programming is a tool for solving some combinatorial optimization problems. In this paper, we deal with combinatorial optimization problems on finite topologies. We use the binary representation of the sets to characterize finite topologies as the solutions of a Boolean quadratic system. This system is used as a basic model for formulating other types of topologies (e.g. door topology and $T_{0}$-topology) and some combinatorial optimization problems on finite topologies. As an example of the proposed model, we found that the smallest number $m(k)$ for which the topology exists on an $m(k)$-elements set containing exactly $k$ open sets, for $k=8$ and $k=15$ is 3 and 5 , respectively.
\end{abstract}

Key words and phrases: finite topology, mathematical modeling.

\footnotetext{
${ }^{1}$ Department of Mathematics, Faculty of Mathematical Sciences, Alzahra University, Tehran, Iran

2 Department of Pure Mathematics, Ferdowsi University of Mashhad, Mashhad, Iran

E-mail: e.monabbati@alzahra.ac.ir (Monabbati S.E.), h.torabi@um.ac.ir (Torabi H.)
}

\section{INTRODUCTION}

Integer programming is a useful tool for solving combinatorial optimization problems. The first step in this solving process is building a mathematical model from the combinatorial problem. Some modeling techniques have been discussed in $[5,6]$ and the references therein. There are some combinatorial optimization problems on finite topological spaces. The most wellknown of them concerns the number of finite topologies on $n$ elements, $T(n)$. K. Ragnarsson and B.E. Tenner in [4] state that this problem can be refined by counting $T(n, k)$, the number of topologies on $n$ elements having $k$ open sets. They also point out the number $T(n)$ is asymptotically equal to $T_{0}(n)$, the number of $T_{0}$-topologies on $n$ elements. Proving the equivalence between $T_{0}$-topologies and cellular complexes, V.A. Kovalevsky [1] solves some contradictions or paradoxes in image analysis. The other combinatorial problem arises in finite topological spaces introduced by K. Ragnarsson and B.E. Tenner [4] is to find the smallest positive integer $m(k)$ such that there exists a topology on $m(k)$ elements having $k$ open sets.

$\mathrm{y} \Delta \mathrm{K} 515.12,519.854 .3$

2010 Mathematics Subject Classification:54A99, 90C10, 90C27.

Corresponding author: Torabi $\mathrm{H}$. 
In this paper, we consider the mathematical modeling of combinatorial problems on finite topological spaces. As well as modeling problems related to obtaining $m(k), T_{0}(n), T(n, k)$, the mathematical models of other topological concepts such as connected components, door topologies, connected topologies, etc. are also presented.

In Section 2 we recall some essential definitions and lemmas. In Section 3, first, we propose a quadratic zero-one system for general finite topological spaces and, then, we use it as the feasible region of models related to the other types of finite topologies. In Section 4, using the notion of topological basis we offer some model improvements which reduce the size of the model in constraints. Then we present an algorithm for the problem of counting the number of finite topologies on an $n$-set. In Section 5 we exhibit an example of combinatorial problems, which are solved by this mathematical programming approach.

\section{DEFINITIONS}

In this section, some basic definitions are presented. Many of them are well-known in the literature [3] and presented here for the sake of completeness. We begin by the definition of a topology.

Definition 1. Let $X$ be a nonempty set. Denote by $P(X)$ the set of all subsets of $X$. A subset $\tau$ of $P(X)$ is called a topology on $X$ if it satisfies the following

(a) $\varnothing, X \in \tau$,

(b) the intersection of finitely many elements of $\tau$ is in $\tau$,

(c) the arbitrary union of elements of $\tau$ is in $\tau$.

The pair $(X, \tau)$ is called a topological space. Obviously, $P(X)$ is itself a topology on $X$ which is called the discrete topology. A set $A \subseteq X$ is said to be open (closed) in $X$ with respect to $\tau$ if $A \in \tau\left(A^{c} \in \tau\right)$. A set which is open and also closed is called a clopen set. A topological space $(X, \tau)$ is called finite if $X$ is a finite set. In this case (c) may be replaced by

(d') finite union of elements of $\tau$ is in $\tau$.

We now introduce some topological concepts which are closely related to our work.

Definition 2. Consider a topological space $(X, \tau)$.

(a) A subset $\beta$ of $P(X)$ is called a topology basis if for any $x \in X$ and $U, V \in \beta$ with $x \in U \cap V$ there is a set $W$ belonging to $\beta$ such that $x \in W$ and $W \subseteq U \cap V$.

(b) $\tau$ is called a door topology if each element of $P(X)$ is either open or close.

(c) $\tau$ has $T_{0}$-property if for each $x, y \in X$ there is an open set in $X$ which contains exactly one of $x$ or $y$. The topology which has the $T_{0}$-property is called $T_{0}$-topology.

(d) Let $A$ be a subset of $X$. The collection $\tau_{A}=\{A \cap G: G \in \tau\}$ is a topology on $A$, which is called the induced topology. With this topology, $A$ is called a subspace of $X$. 
(e) $X$ is called connected if it is not a union of two nonempty disjoint elements of $\tau$. A subset $A$ of $X$ is called a connected set if it is connected as a subspace of $X$. It is easy to see that $X$ is connected if and only if the only its clopen subsets are $X$ and $\varnothing$.

(f) A connected subset $A$ of $X$ is called a connected component if it is not a proper subset of any connected set.

(g) A function $f: X \rightarrow X$ is called continuous with respect to $\tau$ if for any open set $G$, the set $f^{-1}(G)$ is also open.

(h) Let $T$ be a subset of $P(X)$. The intersection of all topologies containing $T$, denoted by $\langle T\rangle$, is called the topology generated by $T$.

We conclude this section by a simple lemma.

Lemma 1. Let $x$ be an integer in $[0, n]$ and $y$ be a binary number which satisfy $\frac{x}{n} \leq y \leq x$. Then $x>0$ if and only if $y=1$.

\section{MODELING}

In this section, we propose mathematical models of various types of topologies introduced in Section 2.

Let $X=\{1, \ldots, n\}$ be an $n$-element set and $P(X)=\left\{\varnothing=G_{0}, G_{1}, \ldots, G_{2^{n}-1}=X\right\}$. Suppose $b_{i} \in\{0,1\}^{n}$ is the binary representation of $G_{i}$, i. e. $b_{i j}=1$ if $j \in G_{i}$ and $b_{i j}=0$ otherwise. There are some bitwise operations on $b_{i}$ 's: "bitwise or", "bitwise and" and "negation". Let $b$ and $c$ are two binary $n$-vectors, then bitwise or, bitwise and of $b, c$ and negation of $b$ are denoted by $b \vee c, b \wedge c$ and $b^{\prime}$, respectively.

Consider the decision variables as follows: $x_{b_{i}}$ is a binary variable which is equal to 1 if $G_{i} \in \tau$ and equal to zero otherwise, $i=0, \ldots, 2^{n}-1$.

Thereinafter, for convenience, we use $i$ instead of $b_{i}$ if there is no ambiguity. Now, any solution $x=\left(x_{0}, x_{1}, \ldots, x_{2^{n}-1}\right)$ of the system

$$
\begin{array}{ll}
x_{i \wedge j} \geq x_{i} x_{j}, & i=1, \cdots, 2^{n}-1, \\
& j=i+1, \ldots, 2^{n}-1, \\
x_{i \vee j} \geq x_{i} x_{j}, & \begin{array}{l}
i=1, \cdots, 2^{n}-1, \\
j=i+1, \ldots, 2^{n}-1,
\end{array} \\
x_{0}=1, & \\
x_{2^{n}-1}=1, & \\
x_{i} \in\{0,1\}, \quad i=0, \cdots, 2^{n}-1,
\end{array}
$$

corresponds to a general topology on the finite set. We denote this system and its solution set by $\operatorname{GFT}(n)$ and $\mathcal{T}$, respectively. It is easy to see that (1), (2) could be replaced by the following inequalities

$$
\begin{array}{ll}
x_{i \vee j}+x_{i \wedge j} \geq 2 x_{i} x_{j}, & i=1, \cdots, 2^{n}-1 \\
& j=i+1, \ldots, 2^{n}-1
\end{array}
$$




\subsection{Modeling special types of topologies}

In this section, we formulate some other types of topologies as a zero-one quadratic system or as a zero-one quadratic optimization problem by adding appropriate equalities/inequalities to $\operatorname{GFT}(n)$.

\subsubsection{Door topology}

By definition $\tau$ is a door topology when exactly one of $G_{i}$ or $G_{i}^{c}=G_{i^{\prime}}$ is in $\tau$. Thus, by adding the constraints $x_{i}+x_{i^{\prime}}=1$, for $i=1, \ldots, 2^{n}-1$ to $G F T(n)$, the door topology's model is at hand.

\subsection{2 $\quad T_{0}$-Topology}

Let $i, j \in X$. The value of $\left(b_{k i}-b_{k j}\right)^{2}$ is equal to 1 if and only if $b_{k i} \neq b_{k j}$. Equivalently $\left(b_{k i}-b_{k j}\right)^{2}=1$ if and only if either $i \in G_{k}$ or $j \in G_{k}$. Thus the sum $\sum_{k=0}^{2^{n}-1} x_{k}\left(b_{k i}-b_{k j}\right)^{2}$ is the number of elements of the topology which contains exactly one of $i$ or $j$. Hence, the inequalities

$$
\sum_{k=0}^{2^{n}-1} x_{k}\left(b_{k i}-b_{k j}\right)^{2} \geq 1, \quad i=1, \ldots, n-1, j=i+1, \ldots, n,
$$

guarantee $T_{0}$-property. We therefore add the inequality (4) to $\operatorname{GFT}(n)$ to achieve a $T_{0}$-topology.

\subsubsection{Topologies for which the induced topology on a fixed subset $A$ (the subspace topol-} ogy on $A$ ) have the $T_{0}$-property

Clearly, $A$ has the $T_{0}$-property if and only if for any two elements of $A$, there is an open set in $X$, contains exactly one of them. Similar to (4) it suffices to add the following constraints

$$
\sum_{k=0}^{2^{n}-1} x_{k}\left(b_{k i}-b_{k j}\right)^{2} \geq 1, \quad i, j \in A .
$$

\subsubsection{Generated topology by a subset $T$ of $P(X)$}

It is easy to see that $\langle T\rangle$ is the smallest topology containing $T$. Thus the following nonlinear integer program gives the generated topology by $T$

$$
\min \sum_{i=0}^{2^{n}-1} x_{i} \quad x_{i}=1, \quad i: G_{i} \in T \quad x \in \mathcal{T}
$$

\subsubsection{Topology $\tau$ for which a given function $f: X \rightarrow X$ is continuous with respect to $\tau$}

Let $f: X \rightarrow X$ be an arbitrary function. We desire to find topology $\tau$ for which $f$ is continuous. Let $F=\left(f_{i j}\right)$ where $f_{i j}=1$ if $f(j)=i$ and $f_{i j}=0$ otherwise. We have

$$
f^{-1}(G)=\left(\max _{i \in G} f_{i 1} \max _{i \in G} f_{i 2} \quad \ldots \quad \max _{i \in G} f_{i n}\right),
$$


since if $b$ is the binary representation of $f^{-1}(G)$, then $k \in f^{-1}(G)$ if and only if $b_{k}=1$. On the other hand $\max _{i \in G} f_{i k}=1$ if and only if at least one of $f_{i k}$ s equal to one, equivalently if and only if there is an $i \in X$ such that $f(i)=k$.

We wish $f^{-1}\left(G_{i}\right)$ in $\tau$, for each $i$. This is attained by the following equations

$$
x_{f_{i}^{-1}}=1, \quad i=1, \ldots, 2^{n}-1,
$$

where $f_{i}^{-1}$ is the binary representation of $f^{-1}\left(G_{i}\right)$.

\subsubsection{Connected topology}

Adding the inequalities

$$
x_{i}+x_{i^{\prime}} \leq 1, \quad i=1, \ldots, 2^{n-1}-1,
$$

implies that for any nonempty proper subset $A$ of $X$, at most one of $A$ and $A^{c}$ is in $\tau$, and by definition, we have a connected topology.

\subsubsection{Topology with the smallest number of connected components}

Suppose some types of topologies are modeled as a mathematical program say (MP). By theorem below one can easily show that including objective function $\sum_{i} x_{i} x_{i^{\prime}}$ to (MP), will result in topology which has the smallest number of connected components.

Theorem 1. Let $(X, \tau)$ be a finite topological space. Then $A \subseteq X$ is clopen if and only if $A$ is a union of some connected components of $X$. Thus, if $F$ is the number of connected components of $X$ then $X$ has exactly $2^{F}$ clopen subsets.

Proof. Let $G_{1}, G_{2}, \ldots, G_{p}$ are the connected components of $X$. Since $X$ is the union of its connected components, then $G_{j}^{c}=\bigcup_{i \neq j} G_{i}$. Therefore $G_{j}^{c}$ is closed, since $G_{i}$ 's are closed by Theorem 1 [2, p. 139]. Thus $G_{j}$ is clopen and it proves that an arbitrary union of connected components is a clopen set.

Conversely, let $A$ be a clopen subset of $X$. We have $X=A \cup A^{c}$. So by Theorem 1 in $\left[2\right.$, p. 131] $G_{i}$ entirely belongs to $A$ or $A^{c}$. On the other hand $X=\cup_{i} G_{i}$ thus

$$
A=\bigcup\left(A \cap G_{i}\right)=\bigcup_{G_{i} \cap A \neq \varnothing}\left(A \cap G_{i}\right)=\bigcup_{G_{i} \cap A \neq \varnothing} G_{i} .
$$

\subsubsection{The topology in which nonempty open sets have at least $k_{1}$ elements and nonempty} closed sets have at least $k_{2}$ elements, $0 \leq k_{1}, k_{2} \leq n$

Let $G$ be any nonempty proper open set. Then $G$ and $G^{c}$ have at least $k_{1}, k_{2}$ elements, respectively. On the other hand $\left|G^{c}\right|=n-|G|$, where $|A|$ denotes number of elements in $A$. Therefore $k_{1} \leq|G| \leq n-k_{2}$. The last inequality is achieved by adding the following equations to $\operatorname{GFT}(n)$

$$
x_{i} k_{1} \leq x_{i} \sum_{j=1}^{n} b_{i j} \leq x_{i}\left(n-k_{2}\right), \quad i=1, \ldots, 2^{n}-1
$$




\subsection{Some combinatorial optimization problems on finite topologies}

This section concerns two combinatorial optimization problems on finite topologies. First, we propose a formulation for computing $m(k)$, the smallest positive integer such that there exists a topology on $m(k)$ elements having $k$ open sets. Then, we propose an algorithm for computing the number $T(n)$ of topologies on an $n$-set.

\subsubsection{Computing $m(k)$}

Topologies with exactly $k$ open sets could be handled by including the equation

$$
\sum_{i=0}^{2^{n}-1} x_{i}=k
$$

to $\operatorname{GFT}(n)$.

K. Ragnarsson and B.E. Tenner in [4] prove that $m(k) \leq \frac{4}{3} \log _{2} k+2$. In addition it's clear that $m(k) \geq \log _{2} k$. Let $n=\left\lfloor\frac{4}{3} \log _{2} k\right\rfloor+2$ and $l=\left\lceil\log _{2} k\right\rceil$. We use the following theorem to formulate the problem as an integer program.

Theorem 2. Suppose $\tau, \tau^{\prime}$ are topologies on

$$
X=\{1,2, \ldots, n\} \quad \text { and } \quad X^{\prime}=\{1,2, \ldots, n, n+1, \ldots, m\},
$$

respectively and let $x, x^{\prime}$ are corresponding binary vectors. Then

$$
\sum_{n=0}^{2^{n}-1} 2^{i} x_{i}<\sum_{n=0}^{2^{m}-1} 2^{i} x_{i}^{\prime}
$$

Proof. We have

$$
\sum_{n=0}^{2^{n}-1} 2^{i} x_{i} \leq \sum_{n=0}^{2^{n}-1} 2^{i}=2^{2^{n}}<2^{2^{m}-1}
$$

Since $\{1,2, \ldots, m\} \in \tau^{\prime}$ then $\sum_{n=0}^{2^{m}-1} 2^{i} x_{i}^{\prime} \geq 2^{2^{m}-1}$ and this completes the proof.

As a consequence of Theorem 2, $m(k)$ is the optimal solution of the following nonlinear integer program:

$$
\begin{aligned}
& \operatorname{MM}(k) \quad \min \sum_{i=0}^{2^{n}-1} 2^{i} x_{i} \\
& \begin{array}{ll}
\text { s.t. } \quad x_{i \wedge j} \geq x_{i} x_{j}, \quad & i=1, \cdots, 2^{n}-1, \\
& j=i+1, \ldots, 2^{n}-1,
\end{array} \\
& x_{i \vee j} \geq x_{i} x_{j}, \quad \begin{array}{l}
i=1, \cdots, 2^{n}-1, \\
j=i+1, \ldots, 2^{n}-1,
\end{array} \\
& \sum_{i=0}^{2^{n}-1} x_{i}=k \\
& x_{0}=1 \text {, } \\
& x_{i} \in\{0,1\}, \quad i=0, \ldots, 2^{n}-1 .
\end{aligned}
$$


Note that we exclude the equality $x_{2^{n}-1}=1$, since if $\beta$ is the topology attained by the system then $\beta$ is the topology on $\bigcup_{A \in \beta} A$.

The $\operatorname{MM}(k)$ may not be useful in practice, because in the objective function when $i$ takes the upper bound of the summation, the value of $2^{2^{n}-1}$ is very large even for small values of $n$. We therefore add new variables $y_{j}$ for $j=1, \ldots, n$ and the following constraints

$$
\frac{1}{n} \sum_{i=0}^{2^{n}-1} x_{i} b_{i j} \leq y_{j} \leq \sum_{i=0}^{2^{n}-1} x_{i} b_{i j}, \quad j=1, \ldots, n .
$$

If $\sum_{i=0}^{2^{n}-1} x_{i} b_{i j}>0$ then $x_{i} b_{i k}=1$ for some $0 \leq i \leq 2^{n}-1$ and $k \in X$. Thus by Lemma $1, y_{k}=1$ if and only if $k$ is in some open set. Hence, the sum $\sum_{j=1}^{n} y_{j}$ gives the number of topology elements. Consequently we can use this sum for an objective function of $\operatorname{MM}(k)$ as follows

$$
\begin{aligned}
& \operatorname{MM}(k) \quad \min \sum_{j=1}^{n} y_{j}, \\
& \text { s.t. } x_{i \wedge j} \geq x_{i} x_{j} \\
& i=1, \cdots, 2^{n}-1 \text {, } \\
& j=i+1, \ldots, 2^{n}-1 \text {, } \\
& x_{i \vee j} \geq x_{i} x_{j}, \\
& i=1, \cdots, 2^{n}-1 \text {, } \\
& j=i+1, \ldots, 2^{n}-1 \text {, } \\
& \frac{1}{n} \sum_{i=0}^{2^{n}-1} x_{i} b_{i j} \leq y_{j} \leq \sum_{i=0}^{2^{n}-1} x_{i} b_{i j}, \quad j=1, \ldots, n, \\
& \sum_{j=1}^{n} y_{j} \geq l \\
& \sum_{i=0}^{2^{n}-1} x_{i}=k \\
& x_{0}=1 \text {, } \\
& x_{i} \in\{0,1\} \text {, } \\
& i=0, \ldots, 2^{n}-1 \text {. }
\end{aligned}
$$

We conclude this section with an example.

Example 1. For $k=8$ we have $n=6$ and $l=3$. We solve $M M(k)$ using LINGO software and obtain $m(8)=3$ with topology $\tau_{8}=P(\{1,2,3\})$. Similarly for $k=15$ again $n=6$ but $l=4$ and $m(15)=5$ and the corresponding topology is

$$
\begin{aligned}
\tau_{15}=\{\varnothing,\{2\},\{1,2\},\{3\},\{2,3\},\{1,2,3\},\{4\},\{2,4\},\{1,2,4\}, \\
\{3,4\},\{2,3,4\},\{1,2,3,4\},\{3,4,5\},\{2,3,4,5\},\{1,2,3,4,5\}\} .
\end{aligned}
$$

\subsubsection{Number of finite topologies}

Suppose that any indiscrete topology has at most $2^{n}-t(n)$ elements. While adding the inequality

$$
\sum_{i=0}^{2^{n}-1} x_{i} \leq 2^{n}-t(n)-1
$$


to GFT $(n)$ drops discrete topology from solution space. It may improve solving process (e.g. it decreases the number of nodes in branch and bound method). Using this fact one may provides an algorithm for counting number of topologies on the finite set, $T(n)$.

Let $\mathcal{T}_{k}^{\leq}=\left\{x \in \mathcal{T}: \sum x_{i} \leq k\right\}$.

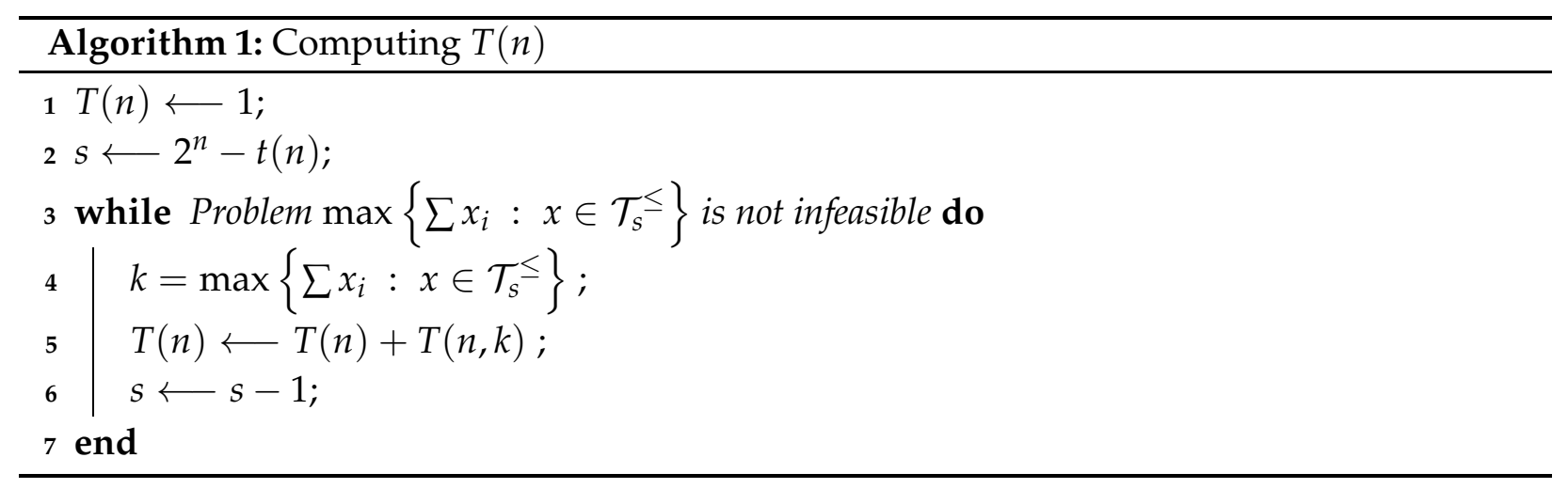
this sum.

Note that $T(n)=\sum_{k=1}^{2^{n}-1} T(n, k)$. In line 4 of the algorithm we omit to compute zero terms of

\section{MOdEL IMPROVEMENT}

In this section, we mention some improvements in models. It is well-known that some properties on topologies could be restricted to a topology basis. Let us consider the problem of the existence of such topologies. For example, is there any $T_{0}$-topology on $n$-set for which a given function $f$ is continuous? To answer such problems we can consider only the topology basis. For this goal it suffices to keep the constraints related to closeness under intersection (constraints (1)) and remove constraints (2) and (3) from GFT $(n)$. By this approach if there is a $T_{0}$-topology for which a given function $f$ is continuous, then we find its basis, else, the model is infeasible.

\section{CONCLUSION}

In this paper, we exhibited mathematical models of some combinatorial problems on finite topologies. As well as modeling problems related to obtaining $m(k), T_{0}(n), T(n, k)$, the mathematical models of other topological concepts such as connected components, door topologies, connected topologies, etc. are also presented. First, we proposed a quadratic zero-one system for general finite topological spaces and then use it as the feasible region of the other types of finite topologies models. We also provided an algorithm for the problem of counting the number of finite topologies on $n$ elements set. It is possible to incorporate models introduced in section 3, to handle another type of problems. For example to answer the question: "is there any door topology $\tau$, for which given function $f$ continuous with respect to $\tau$ ?", it suffices to combine 3.1.1 and 3.1.5. 


\section{REFERENCES}

[1] Kovalevsky V.A. Finite topology as applied to image analysis. Computer vision, Graphics and Image Processing 1989, 46 (2), 141-161. doi:10.1016/0734-189X(89)90165-5

[2] Kuratowski K. Topology. Vol. 2. Academic Press, 1968.

[3] Munkres J.R. Topology. Prentice Hall, 2000.

[4] Ragnarsson K., Tenner B.E. Obtainable sizes of topologies on finite sets. J. Combin. Theory Ser. A 2010, 117 (2), 138-151. doi:10.1016/j.jcta.2009.05.002

[5] Rardin R.L. Optimization in operations research. Prentice Hall, 1997.

[6] Williams H.P. The formulation and solution of discrete optimisation models. In: Appa G., Pitsoulis L., Williams H.P. (eds) Handbook on Modelling for Discrete Optimization. International Series in Operations Research \& Management Science, vol 88. Springer, Boston, MA. doi:10.1007/0-387-32942-0_1

Received 30.05.2019

Монаббаті С.Е., Торабі Х. Математичне моделювання скінченних топологій // Карпатські матем. публ. - 2020. - Т.12, №2. - С. 434-442.

Цілочисельне програмування — це інструмент для вирішення деяких комбінаторних задач оптимізації. У цій роботі ми розглядаємо комбінаторні задачі оптимізації на скінченних топологіях. Ми використовуємо двійкове представлення множин для характеристики скінченних топологій як розв'язків булевої квадратичної системи. Ця система використовується як базова модель для формулювання інших типів топологій (наприклад, топологія дверей та $T_{0}$-топологія) та деяких комбінаторних задач оптимізації на скінченних топологіях. Як приклад запропонованої моделі ми виявили, що найменше число $m(k)$, для якого існує топологія на наборі $m(k)$ елементів, що містить рівно $k$ відкритих наборів, при $k=8$ і $k=15$ - це 3 i 5 вілповіАно.

Ключові слова і фрази: скінченна топологія, математичне моделювання. 